\title{
Introducing PeerXPress for Powder Diffraction
}

It is with great pleasure and anticipation that we announce PeerXPress (PXP) for Powder Diffraction. PeerXPress is an automated manuscript processing system. Authors should now submit their manuscripts online at the following address: http://pdj.peerx-press.org and track the progress of the acceptance process. This editorial will briefly go over some information that will be useful to you when submitting manuscripts to us. You will find revised notes for authors in this issue to help assist you with submitting manuscripts using this system. You can also find them on our website: http://www.icdd.com/resources/pdj/authors.htm. Please read over the instructions carefully and we look forward to your future submissions!

All first time PXP users must register for a new account. From the main login page you will see "New users should register for an account". It will first check to see if you already have one, and if not, will prompt you for contact information. Once you have registered and are logged in, you are ready to submit your manuscript. You will see the prompt to "Submit Manuscript". Follow the prompts to upload your manuscript and figure files. Please note figures must be uploaded separately and not embedded in the manuscript itself. You will be asked to identify the file type as article or figure and check the order of your files. Once you have done this, all the files are combined in to one PDF file and you are asked to review the PDF file to make sure it has all the contents you expect. For help and guidelines, please visit: http://pdj.peerx-press.org/html/pdj/help.html.

I hope you find PeerXPress for PDJ to be a fast and easy tool to submit your manuscripts to us, allowing you to easily track the status of your work and contact us should you need assistance. For editorial inquiries, please contact me at ernst@icdd.com. For technical assistance, please contact pxphelp@aip.org.

Nicole M. Ernst Managing Editor 